\title{
Success rate of using metronidazole paste in pulpal treatment of infected deciduous molars: 6-30 months follow up
}

\author{
Meltem Bakkal ${ }^{1, *}$, Mustafa Sarp Kaya ${ }^{2}$ \\ ${ }^{1,2}$ Assistant Professor, Dept. of Pediatric Dentistry, Bezmialem Vakif University, Istanbul, Turkey
}

*Corresponding Author:

Email: mbakkal@bezmialem.edu.tr

\begin{abstract}
Introduction: The aim of this retrospective study was to evaluate the long term success of using a ready to use metronidazole paste in the pulpal treatment of non-vital deciduous teeth. The assessment was performed by clinical and radiographic records obtained from follow-ups at 6-30 months.

Materials and Methods: Data of 166 children, who had pulpal treatment on their deciduous teeth between April 2013 through June 2016 by a single pediatric dentist, were reviewed for the present study. A pre-treatment history of fistula or swelling, tenderness to percussion, presence of abscess, excessive tooth mobility, radiolucency in the bifurcation and/or periradicular and/or periapical area cases were eligible for inclusion. Finally a total of 42 children with 47 deciduous molars treated with metronidazole paste participated in the study. To determine the success at 6-30 months after treatment, the clinical/radiographic data were evaluated by the principal investigator and a co-investigator. Categorical data association was tested with Chi square test with significance levels at $\mathrm{p}<0.05$ and $95 \%$ confidence intervals.

Result: Success rate of non-vital deciduous teeth was determined as $83 \%$ at 6- 30 months after treatment and it was neither associated with gender $(\mathrm{p}=0.445)$, first/ second molar $(\mathrm{p}=0.692)$ nor maxillary/mandibular molar $(\mathrm{p}=0.853)$.

Conclusion: Keeping a deciduous tooth in the arch is important to improve oral health of children. Metronidazole paste can be suggested to use for the local treatment of pulpal infections following a dental pulp necrosis and their complications in deciduous teeth, instead of performing extractions for them.
\end{abstract}

Keywords: Dental caries, Deciduous molar, Lesion sterilization, Metronidazole paste, Pulpal treatment.

\section{Introduction}

Dental caries is the most prevalent chronic disease in children and tends to progress rapidly. Depending on the severity of caries, pulp interventions are indicated in deciduous teeth. When the radicular pulp of a deciduous tooth is inflamed, the pulpal treatment choice is pulpectomy. ${ }^{1}$ The conventional procedure of pulpectomy for a deciduous tooth is performed by the removal of all pulp tissue from the area of pulp chamber and root canals. The treatment first requires the elimination of bacterial infection, and then needs the prevention of re-infection of this space. Although an excellent pulpectomy in deciduous teeth is claimed to be not achievable due to the accessory canals of deciduous teeth, it is worth a try to keep a deciduous tooth as a guide for the eruption of a permanent tooth. ${ }^{2,3}$ Additional to the difficulties proceeding from morphological alterations of deciduous teeth, challenging behaviour management of children is also a reason in the failure of conventional pulpectomy procedure which is long and complicated. ${ }^{4}$ Modifications to conventional pulpectomy procedure has been proposed to increase the success of root canal treatment in deciduous teeth. Also a simple and less time consuming technique is needed to perform endodontic treatment of infected deciduous teeth instead of their extraction by dental practitioners.

In 2004, lesion sterilization and tissue repair (LSTR) therapy was developed as a technique that allows disinfection of pulpal and periradicular lesions via using corticostreoids and/or antibiotics. ${ }^{5-7}$ Due to the fact that LSTR has no mechanical instrumentation which prevents the unnecessary enlargement of root canals and irritation of periapical tissues, the technique is also introduced as Non Instrumental Endodontic Treatment (NIET). ${ }^{8}$ The procedure includes only an enlargement of the root canal orifice to deliver the medicament. But the residual inflamed pulp tissue was reported as the possible reason for internal root resorption..$^{9}$ In a study done by Prabhakar et al., this technique was used with radicular pulp extirpation and higher success was found in the extirpated pulp group. ${ }^{10}$

The infection of root canal system is a polymicrobial infection and a combination would be needed to address the diverse flora encountered. Several combinations of antibacterial drugs are placed to eliminate the bacteria from deep layers of infected root canal systems. Among these, a combination of Metronidazole and ciprofloxacin plus minocycline (Triple Antibiotic paste) has been popularly used with varying success. ${ }^{11,12}$ However tooth discoloration come from tetracycline family has been reported as a disadvantage of this mixture. ${ }^{13,14}$ Several factors such as adequate concentration of drug, type of infection, diffusion ability of paste, biocompatibility and systemic toxicity of material influence the selection of proper medicaments to sterilize the root canals. ${ }^{5,15}$

Ciprofloxacin is effective against gram negative organisms. Meanwhile minocycline is a broad spectrum antimicrobial agent which is effective against both 
gram negative and gram positive microorganisms and spirochetes. Metronidazole is a nitroimidazole compound used particularly for anaerobic cocci, bacilli (both gram positive and gram negative) and a few protozoa. ${ }^{16}$ Although there have been many studies showing the combination of these 3 antibiotics on the effect of endodontic lesions, data on using metronidazole singly in deciduous teeth is limited. ${ }^{15,17}$ In a clinical study by Kargul et al., $75 \%$ overall success with a 24 mean follow-up duration was reported in pulpectomies of deciduous teeth after using freshly prepared metronidazole dressing as an intracanal medicament which was prepared with mixing metronidazole tablets with sterile saline. ${ }^{18}$

The aim of this retrospective study was to evaluate the success rate of placing "ready to use" metronidazole paste on pulp chambers after the extirpation of pulp tissue from root canals in the first visit of pulpal treatment for non-vital deciduous molars which were then obturated with ZOE in the second visit. The assessment was performed by clinical and radiographic data at 6-30 months.

\section{Materials and Methods}

This study was completed by the Ethics Committee of Bezmialem Vakif University (Protocol Number: 71306642-050.01.04-). Hundred and sixty six records were evaluated which had a pulpal treatment between April 2013 through June 2016 (3 years) in the Pediatric Dentistry Department of Bezmialem Vakif University by the same clinician. From the 166 records who had undergone endodontic treatment, 89 children treated with a ready to use metronidazole paste (Grinazole, Septodont, Paris, France) were investigated first, and finally a total of 42 children with 47 treated deciduous molars fulfilling the inclusion criteria were included in the study.

The inclusion and exclusion criteria: Non-vital deciduous molars treated with metronidazole paste which had any history of fistula or swelling, tenderness to percussion, presence of abscess, excessive tooth mobility, radiolucency in the bifurcation and/or periradicular and/or periapical area at the beginning of the treatment were included to the study. The subjects with any systematic conditions, children who received antibiotic treatment during the performance of pulpectomy, the teeth with any morphological anomalies were excluded from the study. Teeth had initial radiographs dating back more than 6 months at start of the treatment and children who had their last follow-up records dating back more than 6 months at start of this retrospective study were also excluded from the study.

Treatment protocol for NIET technique performed with pulp extirpation: Local anesthesia was usually not required during the procedure of most teeth because of pulp tissue necrosis. Caries were removed prior to pulpal access. Following the removal of all pulp tissue from the pulp chamber, a barbed broach (tire nerf; yellow, red or blue in colors; proper size to root canals) was used to extirpate the pulp from the root canals. Irrigation with $2.5 \%$ sodium hypochlorite solution $(\mathrm{NaOCl})$ was performed from the canal orifice, with no pressure, to improve the debridement and to control the hemorrhage. Subsequent to rinsing, cotton pellets were used for drying. Then metronidazole paste was applied to pulp chamber with a cotton pellet before the placement of temporary filling material (Zinc oxide eugenol cement, (ZOE) Cavex, Haarlem, Holland). Four days later, after removing the metronidazole paste by irrigation with $\mathrm{NaOCl}$, the pulp chamber were obturated with ZOE cement. Then a glass ionomer cement (3M ESPE, Ketac Bond Glass Ionomer Base Material) was used as a cavity liner and then final restoration was performed with compomer resin (Dyract Extra, Dentsply, Germany). From then on, children were asked to return at every 6 months for clinical and radiographic follow-ups as routine.

Assessment criteria and statistical analysis: The principal investigator and a co-investigator evaluated the subjects and a consensus was agreed upon for each result. The treatments were recorded as successful if they exfoliated naturally and had no symptoms of failure. The criteria for rating as failed clinically were; presence of spontaneous pain, soft tissue pathology or fistula and/or adjacent tissue inflammation such as symptoms of abscess or swelling, secondary caries and/or failure in restoration, tenderness to percussion, higher mobility when compared to symmetric tooth. The radiographic evaluation was performed and rated as failed with the following criteria; abnormal radiolucency of filling material, secondary caries or failure in restoration radiographically, pathological root resorption such as external and/or internal root resorption, pathological radiolucency caused by interradicular and/or periapical bone destruction, early root resorption when compared to symmetric tooth.

Categorical data association was tested with Chi square test at $\mathrm{p} \leq 0.05$ significance. All descriptive statistics and tests were performed using SPSS 20.0.

\section{Results}

A total of 42 children (19 boys, 23 girls; $82.04 \pm$ 21.5 months old) with 47 treated deciduous molars participated in the study. The clinical and radiographic records were used to evaluate the success rate of these treated deciduous molars. Nineteen maxillary deciduous molars ( 7 first molars, 12 second molars) and 28 mandibular deciduous molars (10 first molar, 18 second molar) were evaluated by two investigators. The distributions of mandibular and maxillary deciduous molars according to follow-up time are given in Table 1.

Clinical and radiographical data were evaluated and a total success rate of these non-vital deciduous 
teeth was determined as $83 \%$ at 6- 30 months after treatment.

The records of clinical examination showed that none of 47 teeth were symptomatic with spontaneous pain at any time of follow-ups, even though some had periradicular radiolucency at the start of the treatment (Fig. 1). However the clinical record showed that 2 teeth had soft tissue swelling and 4 teeth were tender on percussion. Pathological mobility was seen in 3 deciduous molars which were rated as failed, too. According to radiographical evaluation, pathological root resorption was observed in 9/47 of the molars and also $9 / 47$ of them had pathological lesions which were examined radiographically. Only a single tooth was diagnosed with clinical secondary caries whereas 5 teeth had radiological caries, both were not associated with failure at any follow up time $(\mathrm{p} \leq 0.05)$. None of the root canals were observed with any filling material abnormality Table 2 .

In addition, no overfilled root canals were seen in radiographs, whereas underfilled canals were common as NIET technique does not include an instrumentation to enlarge the root canals. Since the NIET technique was performed only with pulp extirpation in the present study, all of the root canals was underfilled and this condition was not associated with failure $(\mathrm{p} \leq 0.05)$ Fig. 2.

Success or failure outcomes were not associated with follow up time Table 3 . In addition, success rates were neither associated with gender $(p=0.445)$, first/ second molar $(p=0.692)$ nor maxillary/mandibular molar $(\mathrm{p}=0.853)$. Success rate was $16 / 19(84.2 \%)$ for maxillary, 23/ 28 (82.1\%) for mandibular molars, 15/17 $(88.2 \%)$ for first molars, $24 / 30(80 \%)$ for second molars.

Table 1: Number of teeth according to follow up time

\begin{tabular}{|l|c|c|c|c|}
\hline & No. of teeth & 6-12 months & 12-24 months & 24-30 months \\
\hline Decidious maxillary molars & $19(40.4 \%)$ & $5(10.6 \%)$ & $4(8.5 \%)$ & $10(21.3 \%)$ \\
\hline Decidious mandibular molars & $28(59.6 \%)$ & $10(21.3 \%)$ & $7(14.9 \%)$ & $11(23.4 \%)$ \\
\hline Total & $47(100 \%)$ & $15(31.9 \%)$ & $11(23.4 \%)$ & $21(44.7 \%)$ \\
\hline
\end{tabular}

Table 2: Clinical and radiographic evaluation data according to follow up time

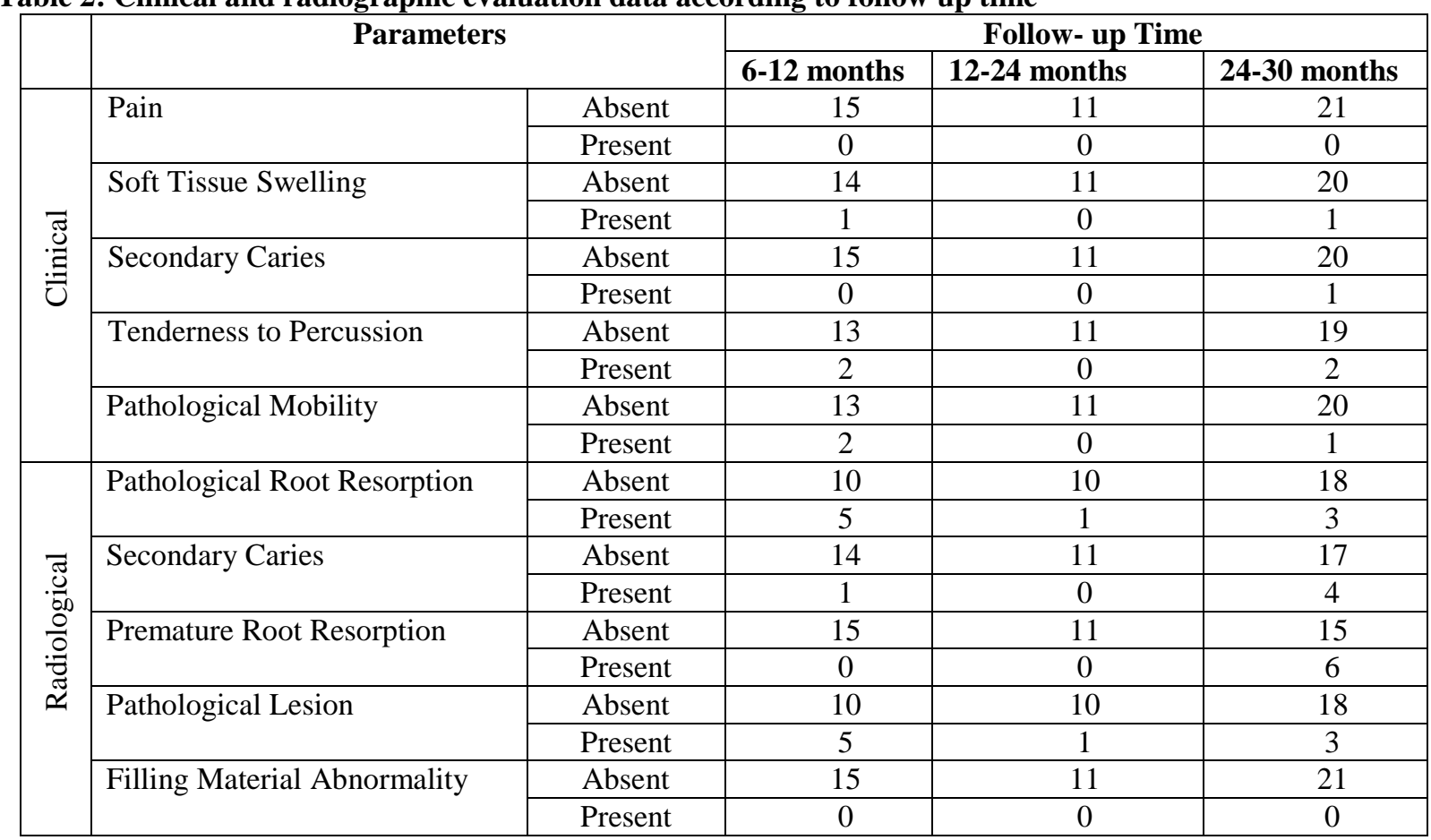


Table 3: Success or fail outcome according to follow up time

\begin{tabular}{|l|c|c|c|l|l|}
\hline & \multicolumn{3}{|c|}{ Follow- up Time } & \multirow{2}{*}{ P value } & \\
\cline { 2 - 5 } & $\mathbf{6 - 1 2}$ months & $\mathbf{1 2 - 2 4}$ months & $\mathbf{2 4 - 3 0}$ months & Total & \\
\hline Success & 14 & 10 & 15 & 39 & \\
& $(29.8 \%)$ & $(21.3 \%)$ & $(31.9 \%)$ & $(83.0 \%)$ & \\
Failure & 1 & 1 & 6 & 8 & \\
& $(2.1 \%)$ & $(1.1 \%)$ & $(12.8 \%)$ & $(17 \%)$ & \multirow{2}{*}{0.164} \\
\cline { 1 - 5 } Total & 15 & 11 & 21 & 47 & \\
& $(31.9 \%)$ & $(23.4 \%)$ & $(44.7 \%)$ & $(100 \%)$ & \\
\hline
\end{tabular}
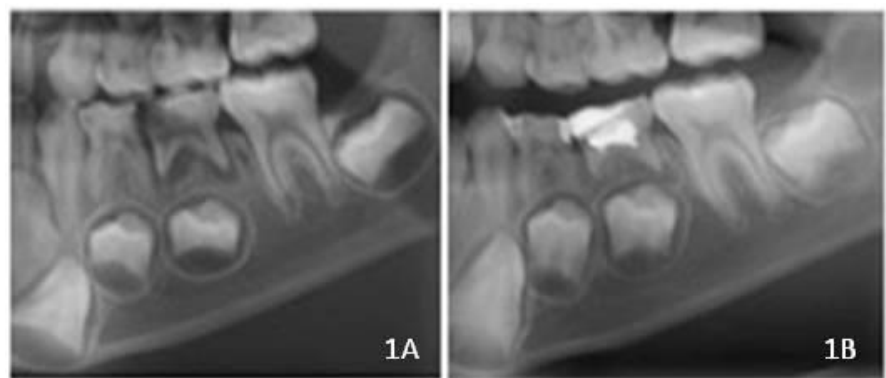

Fig. 1A: Before the treatment of the mandibular left deciduous second molar, 1B: Twelve months after treatment showing healing in periradicular radiolucency without any symptoms such as pain, tenderness to percussion, and pathological mobility
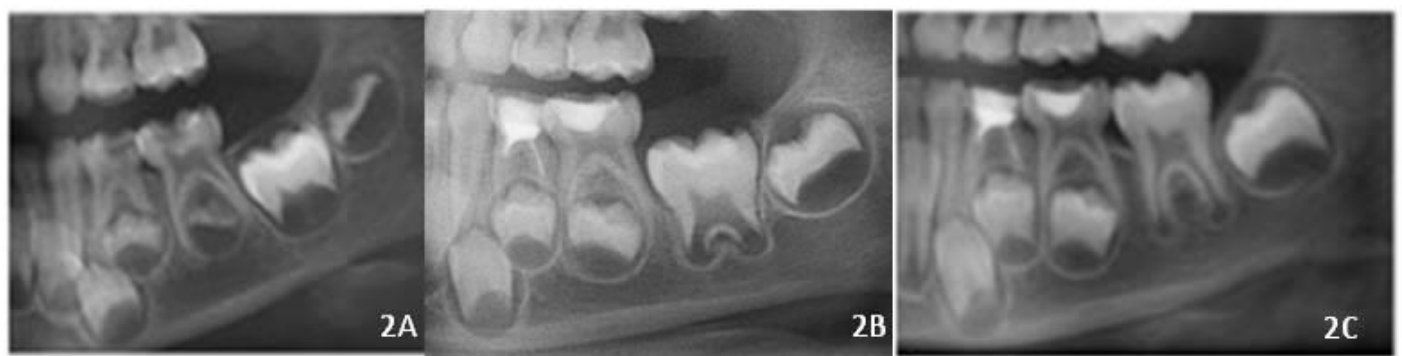

Fig. 2A: Before the treatment of mandibular left deciduous first molar, 2B; Twelve months after treatment, 2C; Thirty months follow up showing a successful prognosis

\section{Discussion}

Preservation of deciduous teeth is the best space maintainer for its successor. Pulpectomy is one of the suggested pulpal treatment choices for deciduous teeth to keep them in the arch. ${ }^{1}$ Unfortunately, many dentists in public prefer extraction of deciduous teeth instead of performing an endodontic treatment, because pulp therapy in deciduous teeth is difficult because of the reasons like differences in morphology, complexity of root canals, need for longer chair time especially for younger children and desired timely resorption of root canal filling materials. ${ }^{9}$

NIET technique presents several advantages such as time saving, no instrumentation and lesion sterilization. But the data about the success of this simplified technique compared to conventional procedure is insufficient. In a study of Trairatvorakul et al. the clinical success rate in NIET performed with 3 Mix was reported as $75 \%$ at 6 months follow up period. ${ }^{9}$ They also reported higher success in second deciduous molars then first molars. ${ }^{9}$ But the researchers pointed that NIET with $3 \mathrm{Mix}$ cannot replace the conventional pulpectomy due to its declining clinical success at 24- 27 months. In this regard we aimed to show whether lesion sterilization with metronidazole paste singly give a long-term success in the treatment of infected deciduous teeth.

Trairatvorakul et al. suggested that the residual inflamed pulp tissue might be the reason of internal root resorption observed in NIET performed deciduous teeth at one year follow-up..$^{9}$ In addition, the combination of ciprofloxacin, metronidazole, and minocycline was used in the study of Prabhakar et al. who reported that the group; NIET performed with radicular pulp extirpation showed higher success than the group had only coronal pulp removal. ${ }^{10}$ Conventional endodontic treatment depends on the elimination of anaerobic microorganisms, such as Porphyromonas endodontalis, Porphyromonas gingivalis, Prevotella intermedia, Prevotella melaninogenica, Fusobacterium nucleatum and other Fusobacterium species which are present in infected pulp tissue. ${ }^{18}$ Mechanical enlargement of root 
canals and removal infected dentine from root canals in conventional endodontic treatment may not remove all the bacteria in these areas, thus the use of irrigation solutions and intracanal medicaments are aimed to inactivate the remaining bacteria. In NIET the root canals are not enlarged and a mixture of antibacterial drugs are used to disinfect the root canals. In a previous study, Pinky et al reported $90 \%$ success at 12 months after using a mixture of ciprofloxacin, metronidazole and minocycline (3Mix) with NIET in non vital teeth. ${ }^{15}$ The therapeutic effects of local application of antibiotics and/or corticosteroids can be proposed for disinfection of pulp tissue without the potential risk of its adverse systemic reactions. However it should be taken into account that antibiotics are restricted to be used in the patients who are non-allergic to the medicine and/or its family (imidazole) and/or any of the other ingredients of this medicine. The amount of drug should be enough for adequate sterilization and not to damage the host cells. Using polyantibiotic paste containing penicillin has a decrease popularity because of the potential sensitization of patients. Causing bacterial resistance is another disadvantage of polyantibiotic pastes, in addition to discoloration of crown brought by tetracycline family. Polyantibiotic pastes which are produced by mixing of preferred antibiotics with equal amounts and then glycol is added to produce a creamy form, need the knowledge of method for preparation and storage in air-tight containers. In this study we preferred using a ready to use metronidazole paste singly to minimize the disadvantages of polyantibiotic pastes such as bacterial resistance and sensitization to the drugs, preparation and storage of the paste.

In a similar study by Kargul et al. using metronidazole tablets for canal dressing before the obturation with ZOE yielded an overall $75 \%$ success with a 24 mean follow-up duration of infected deciduous teeth. ${ }^{18}$ In the lights of these outcomes, the present study aimed to reveal the success rate of NIET with ready to use metronidazole paste in infected deciduous teeth at 6-30 months. The overall success rate at a period of 6-30 months was found $83 \%$ in our study. Although our method is similar to Kargul et al. their lower success rate may be due to uncertain mixing proportions of freshly mixed metronidazole tablets and the physicochemical property of saline as a vehicle used in their study. ${ }^{18}$

Calcium hydroxide $\left(\mathrm{Ca}(\mathrm{OH})_{2}\right)$, ZOE, KRI paste and $\mathrm{ZOE} /$ Iodoform paste are the common root canal filling materials used in deciduous teeth. In a study by Trairatvorakul et al. comparing ZOE vs. Iodoform/ $\mathrm{Ca}(\mathrm{OH})_{2}$ pulpectomies reported similar overall success (85\% and $89 \%$ respectively) at 12 months. ${ }^{19} \mathrm{ZOE}$ was preferred over calcium hydroxide in the present study due to its better compatible to natural root resorption and its viscous form which prevents overfilling to the periapex. In addition, $\mathrm{ZOE}$ has strong antibacterial effects against endodontic microbiota due to consisting eugenol which has been reported to exhibit this effect rather than zinc oxide. ${ }^{2}$

The prefabricated pediatric crowns (PPC) are recommended for the final restoration in pulpally treated primary teeth. On the other hand, adhesive and amalgam restorations have also been reported acceptable even their microleakage performance is behind PPC. ${ }^{20,21}$ In recent years, compomer resins are also recommended as an alternative restorative material to amalgam in primary molars. ${ }^{22}$ A 3-year clinical follow up study showed that the success rate of compomer resins preferred as final restoration material after primary molar pulpectomy (20 teeth) was $95.0 \%$; while the success rates of Stainless Steel Crowns (11 teeth), amalgam (12 teeth) and Glass Ionomer Cements (9 teeth) were $90.9 \%, 83.8 \%$ and $100 \%$ respectively. ${ }^{23}$ In the present study compomer resin was used for the final restorations and any diagnosed secondary caries due to microleakage of compomer restorations were not found related to the failure of performed endodontic treatments.

\section{Conclusion}

Metronidazole paste can be suggested for local treatment of root canal infections following a dental pulp necrosis and their complications. The risk of drug interaction can be neglected because the administered dose for the treatment of a single tooth is limited. In addition to its safety, a ready to use paste is more practical to place it into root canals due to its creamy form, than freshly mixed intracanal medicament including metronidazole tablet and sterile saline. Furthermore, it is suggested that one of the main factors responsible from failure might be associated with uncertain mixing proportions of freshly mixed metronidazole paste. A ready to use paste could facilitate increased efficacy of metronidazole in necrosed primary teeth.

\section{Acknowledgements}

Authors would like to thank Busra Tekin, Busra Selen Borklu, Ozge Colak who were the fourth year students at Faculty of Dentistry, Bezmialem Vakif University during the academic term of 2015-2016 and Muge Yavas who was a research assistant at the Department of Pediatric Dentistry in 2016 for their contribution in the management of follow-up appointments.

\section{Conflict of Interest: None}

\section{References}

1. Smail-Faugeron V, Courson F, Durieux P. Pulp treatment for extensive decay in primary teeth. Cochrane Database Syst Rev 2014: CD003220.

2. Harini Priya M, Bhat SS, Sundeep Hegde K. Comparative evaluation of bactericidal potential of four root canal filling materials against microflora of 
infected non-vital primary teeth. J Clin Pediatr Dent 2010;35:23-9.

3. Brosnan MG, Natarajan AK, Campbell JM. Management of the pulp in primary teeth--an update. The New Zealand Dent J 2014;110:119-23.

4. de Deus MouraLde F, de Lima Mde D, Lima CC, Machado JI, de Moura MS, de Carvalho PV. Endodontic Treatment of Primary Molars with Antibiotic Paste: A Report of 38 Cases. J ClinPediatr Dent 2016;40:175-77.

5. Jaya AR, Praveen P, Anantharaj A. In vivo evaluation of lesion sterilization and tissue repair in primary teeth pulp therapy using two antibiotic drug combinations. $J$ Clin Pediatr Dent 2012;37:189-91.

6. Aboujaoude S, Noueiri B, Berbari R. Evaluation of a modified Pulpotec endodontic approach on necrotic primary molars: a one-year follow-up. Eur J Paediatr Dent 2015;16:111-14.

7. Takushige T, Cruz EV, Asgor Moral A. Endodontic treatment of primary teeth using a combination of antibacterial drugs. Int Endod $J$ 2004;37:132-38.

8. Burrus D, Barbeau L and Hodgson B. Treatment of abscessed primary molars utilizing lesion sterilization and tissue repair: literature review and report of three cases. Pediatr Dent 2014;36:240-44.

9. Trairatvorakul C and Detsomboonrat P. Success rates of a mixture of ciprofloxacin, metronidazole, and minocycline antibiotics used in the noninstrumentation endodontic treatment of mandibular primary molars with carious pulpal involvement. Int $J$ Paediatr Dent 2012;22:217-27.

10. Prabhakar AR, Sridevi E, Raju OS. Endodontic treatment of primary teeth using combination of antibacterial drugs: an in vivo study. J Indian Soc Pedod Prev Dent 2008;26(1):S5-10.

11. Hoshino E, Kurihara-Ando N, Sato I. In-vitro antibacterial susceptibility of bacteria taken from infected root dentine to a mixture of ciprofloxacin, metronidazole and minocycline. Int Endod $J$ 1996;29:125-30.

12. Sato T, Hoshino E, Uematsu H. In vitro antimicrobial susceptibility to combinations of drugs on bacteria from carious and endodontic lesions of human deciduous teeth. Oral Microbiol Immunol 1993;8:17276.
13. Lenherr P, Allgayer N, Weiger R. Tooth discoloration induced by endodontic materials: a laboratory study. Int Endod J 2012;45:942-49.

14. Kim JH, Kim Y, Shin SJ, Tooth discoloration of immature permanent incisor associated with triple antibiotic therapy: a case report. $J$ Endod 2010;36:1086-91.

15. Pinky C, Shashibhushan KK, Subbareddy VV. Endodontic treatment of necrosed primary teeth using two different combinations of antibacterial drugs: an in vivo study. J Indian Soc Pedod Prev Dent 2011;29:121-27.

16. Raju SM, Yadav SS, Kumar MS. Revascularization of immature mandibular premolar with pulpal necrosis - a case report. J Clin Diagn Res 2014;8:ZD29-31.

17. Panzarini SR, Souza V, Holland R. Association of calcium hydroxide and metronidazole in the treatment of dog's teeth with chronic periapical lesion. J Appl Oral Sci 2006;14:334-40.

18. Kargul B, Kadir T. The antibacterial effects of ornidazole on primary molars with infected pulps. Chemother 2001;47:203-07.

19. Trairatvorakul C, Chunlasikaiwan S. Success of pulpectomy with zinc oxide-eugenol vs calcium hydroxide/iodoform paste in primary molars: a clinical study. Pediatr Dent 2008;30:303-08.

20. Davina AB. A conservative approach to the pulpotomy in primary teeth. J Clin Pediatr Dent 1998;22:103-105.

21. Guelmann M, Bookmyer KL, Villalta P, Garcia Godoy F. Microleakage of restorative techniques for pulpotomized primary molars. J Dent Child (Chic) 2004;71:209-11.

22. Ertugrul F, Cogulu D, Ozdemir Y. Comparison of conventional versus colored compomers for class II restorations in primary molars: a 12-month clinical study. Med Princ Pract 2010;19:148-52.

23. Sari S and Okte Z. Success rate of Sealapex in root canal treatment for primary teeth: 3-year follow-up. Oral Surg Oral Med Oral Pathol Oral Radiol Endod 2008;105:e93-6.

How to cite the article: Bakkal M, Kaya MS Success rate of using metronidazole paste in pulpal treatment of infected deciduous molars: 6-30 months follow up. J Dent Specialities 2018;6(2):115-120. 